\title{
Wavefront shaping of a Bessel light field enhances light sheet microscopy with scattered light
}

\author{
J. Nylk*a,b, C. Mitchell ${ }^{\mathrm{a}, \mathrm{b}}$, T. Vettenburga ${ }^{\mathrm{a}}$, F. J. Gunn-Moore ${ }^{\mathrm{b}}$, K. Dholakia ${ }^{\mathrm{a}}$ \\ ${ }^{a}$ SUPA, School of Physics and Astronomy, University of St. Andrews, North Haugh, St. Andrews, \\ Fife, KY16 9SS, UK \\ ${ }^{\mathrm{b}}$ School of Biology, University of St. Andrews, North Haugh, St. Andrews, Fife, KY16 9TF, UK
}

\begin{abstract}
Light sheet microscopy has seen a resurgence as it facilitates rapid, high contrast, volumetric imaging with minimal sample exposure. Initially developed for imaging scattered light, this application of light sheet microscopy has largely been overlooked but provides an endogenous contrast mechanism which can complement fluorescence imaging and requires very little or no modification to an existing light sheet fluorescence microscope. Fluorescence imaging and scattered light imaging differ in terms of image formation. In the former the detected light is incoherent and weak whereas in the latter the coherence properties of the illumination source, typically a laser, dictate the coherence of detected light, but both are dependent on the quality of the illuminating light sheet. Image formation in both schemes can be understood as the convolution of the light sheet with the specimen distribution.

In this paper we explore wavefront shaping for the enhancement of light sheet microscopy with scattered light. We show experimental verification of this result, demonstrating the use of the propagation invariant Bessel beam to extend the field of view of a high resolution scattered light, light sheet microscope and its application to imaging of biological super-cellular structures with sub-cellular resolution. Additionally, complementary scattering and fluorescence imaging is used to characterize the enhancement, and to develop a deeper understanding of the differences of image formation between contrast mechanisms in light sheet microscopy.
\end{abstract}

Keywords: Light sheet microscopy, LSM, selective plane illumination microscopy, SPIM, beam shaping, Bessel beam, high resolution, deconvolution

\section{INTRODUCTION}

Light sheet microscopy (LSM) is a powerful, wide-field imaging method that has revolutionized fluorescence imaging in developmental biology and related disciplines ${ }^{1-4}$. In light sheet fluorescence microscopy (LSFM) the illumination takes the form of a thin, planar sheet, created either by rapid one-dimensional scanning of a focused spot or with a cylindrical lens, and fluorescence is collected from all points within the light sheet simultaneously by an objective perpendicular to the illumination with its focal plane aligned with the light sheet. This results in rapid, high contrast, volumetric imaging and minimizes sample exposure, limiting photobleaching and other phototoxic effects, and allows extended time-lapse studies to be carried out on delicate biological specimens ${ }^{2,5,6}$. The properties of LSFM make it a promising candidate for imaging plant growth ${ }^{7}$, recently it was shown that LSM utilizing scattered laser light rather than fluorescence for imaging could have possible applications in plant root imaging ${ }^{8}$ and other fields. This method, referred to as light sheet tomography (LST), can only image samples with relatively low densities of scatterers, as a high density of scatterers results in multiple scattering events that distort the image ${ }^{8}$. LST retains all the advantages of LSFM and light scattering gives the added advantages of being endogenous to the sample, eliminating any need to develop stably fluorescent

*jn78@st-andrews.ac.uk

Three-Dimensional and Multidimensional Microscopy: Image Acquisition and Processing XXI,

edited by Thomas G. Brown, Carol J. Cogswell, Tony Wilson, Proc. of SPIE Vol. 8949, 89490V

(C) 2014 SPIE · CCC code: 1605-7422/14/\$18 - doi: 10.1117/12.2038525

Proc. of SPIE Vol. 894989490 V-1 
specimens, and being linearly proportional to the incident light sheet intensity whereas fluorescence is limited by the emission rate of the fluorescence molecule. Although photobleaching is not an issue for LST, light sheet illumination minimizes sample exposure which may still be important to avoid phototoxic effects ${ }^{9}$ and when combining LST and LSFM modalities if the same source is used for scattering and fluorescence excitation.

Although LSFM and LST have different contrast mechanisms they still share similarities and in both cases, both axial resolution and overall image quality are dependent on the thickness and uniformity of the incident light sheet. There is a trade-off between the axial resolution and the field of view (FoV) over which this resolution is achieved due to the rapid divergence of a tightly focused Gaussian beam ${ }^{10}$. In the case of high axial resolution, the useful FoV becomes very small and the optical sectioning advantages of LSM are lost, increasing sample irradiation, photodamage and exposure time. This issue is common to both LSFM and LST but only in fluorescence imaging has wavefront shaping been applied to overcome the problem. To achieve high axial resolution over a larger FoV in LSFM, schemes utilizing scanned Bessel beam illumination have been used, exploiting the propagation invariance of such light modes ${ }^{11-14}$.

Image formation in LSFM can be understood mathematically as a convolution of the light sheet profile with the sample fluorophore distribution, because of this, attainable axial resolution is determined easily by considering the process in the spatial frequency domain using the axial modulation transfer function (MTF), defined for LSFM as the absolute value of the Fourier transform of a z-axis section of the normalized light sheet intensity profile. For the case of a Gaussian light sheet, this argument reduces to the thickness of the light sheet dictating the axial resolution as broadening of the light sheet with propagation from the focus reduces the magnitude of high spatial frequencies in the MTF. This analysis has shown that while Bessel beam illumination can be used to extend the high resolution FoV in LSFM, the use of such beams also reduces the axial resolution as the magnitude of high spatial frequencies are reduced ${ }^{10}$. When scattered light is imaged, there are added complexities in the greater coherence of the detected light but again, axial resolution in LST is dictated by the light sheet thickness and so the convolution of the light sheet with the sample distribution must play a key role in the image formation process.

We demonstrate that, due to the similarities in the image formation process in LSFM and LST, wavefront shaping techniques already applied in LSFM can be directly transferred and applied to LSM when imaging scattered light. We verify this by demonstrating an extension of the FoV of LST with Bessel beam illumination when imaging microsphere based test targets and three-dimensional cell suspensions. A dual purpose light sheet microscope capable of performing both LSFM and LST is used to make direct comparisons between techniques.

\section{MATERIALS AND METHODS}

\subsection{Light sheet microscopy setup}

A dual purpose light sheet microscope, capable of both fluorescence and scattered light imaging, was constructed as described below. The laser beam (Coherent Verdi V6, 6W 532nm) was passed through a single mode fiber and expanded to fill the active area of a spatial light modulator (SLM, Hamamatsu LCOS X10468-04). Rather than using static phase masks to shape the beam, the use of an SLM allowed rapid switching between Gaussian and Bessel light sheets, minimizing the influence of sample movement between the recording of datasets for different beam types. Wavefront correction $^{15}$ was also implemented using the SLM to eliminate systematic aberrations in the optical train of the illumination pathway, ensuring that all light sheet types used here showed only minimal deviations from their theoretical descriptions. The active area of the SLM is imaged onto an acousto-optic deflector (AOD, Neos AOBD 45035-3) which forms the light sheet by rapid scanning of the laser focus along the y-axis. The AOD scan period ( $100 \mu \mathrm{s})$ is much shorter than the shortest acquisition time in our experiments $(10 \mathrm{~ms})$ such that a uniform intensity light sheet is observed. The AOD aperture is re-imaged onto the back aperture of the water dipping excitation objective (MO1, Nikon CFI Apo 40x/0.8 DIC, working distance 3.5mm). Pinholes are placed at the foci of the relay telescopes between the SLM, AOD and excitation objective to select only the first diffraction orders from the SLM and AOD. Fluorescence and laser scatter was detected on an sCMOS camera (Hamamatsu Orca Flash 4.0) using an orthogonally mounted water dipping objective (MO2, Nikon CFI Apo 40x/0.8 DIC, working distance 3.5mm) and appropriate tube lens. When imaging in fluorescence, an appropriate laser line filter (LLF) was placed in front of the camera. This filter was removed for imaging scattered light. No fluorescence filter was required to reject fluorescence for scattered light imaging as the scattering signal was 
found to be over 100 times stronger than the fluorescence signal,. Both the illumination and detection objectives had their numerical aperture (NA) limited to 0.42. The sample (see Section 2.4 for preparation) was mounted on an xyz piezo-stage (Mad City Labs, Nano-LP200) at $45^{\circ}$ to both excitation and detection objectives to allow precise positioning of the sample with respect to the light sheet and was moved in steps of $200 \mathrm{~nm}$ along the $\mathrm{z}$-axis for the acquisition of volumetric scans. The Cartesian coordinate system referred to for this microscope is defined as follows; the propagation axis of the illumination objective is the $\mathrm{x}$-axis; the AOD scan direction is the $\mathrm{y}$-axis, the propagation axis of the detection objective is the z-axis.

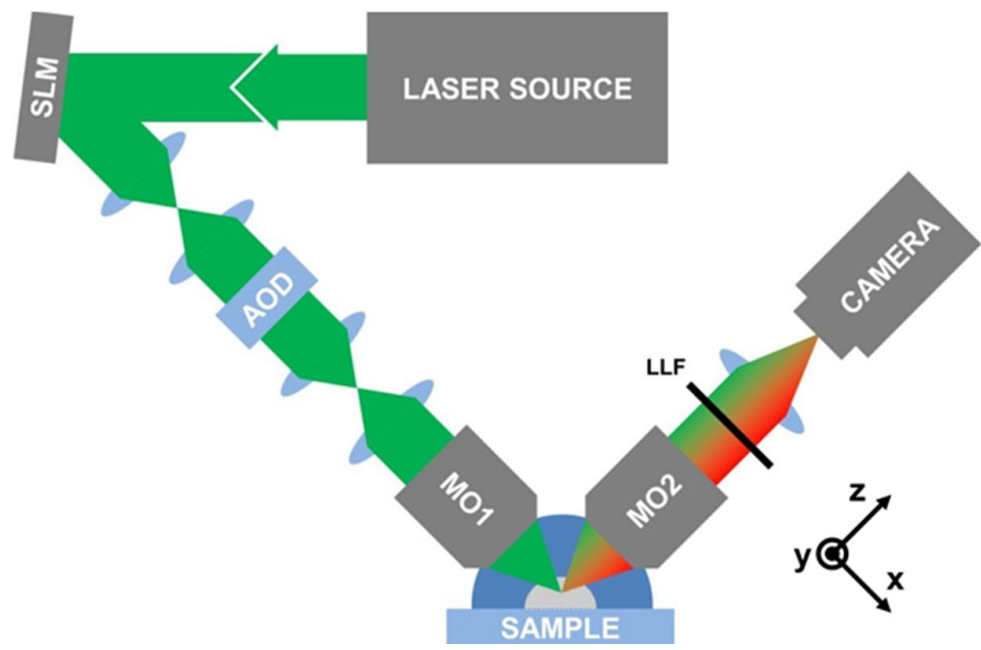

Figure 1. Schematic diagram of LSFM/LST microscope. Only the paths of the first diffraction orders of the SLM and AOD are shown for clarity. See main body for description.

\subsection{Deconvolution}

Axial resolution in LSM is lost when away from the Gaussian beam focus or when using non-Gaussian beams for illumination due to the reduction of spatial frequencies in the MTF, however, this axial resolution loss can be alleviated through the use of computational deconvolution. A one-dimensional Wiener deconvolution along the z-axis was found to be sufficient to restore axial resolution is LSM. A detailed description of the algorithm and its motivation can be found in Section 5.8 of reference 16 .

\subsection{Cell culture}

MCF-10A cells stably expressing red fluorescent protein (RFP) were grown in cell culture flasks (Nucleon T25, Fisher Scientific) under standard conditions $\left(37^{\circ} \mathrm{C}, 5 \% \mathrm{CO} 2\right)$ and in growth medium comprising Dulbecco's modified eagles medium (DMEM/F-12, Sigma Aldrich) supplemented with 5\% (v/v) horse serum (HS, Invitrogen), 20ng/ml epidermal growth factor (EGF, Invitrogen), $0.5 \mu \mathrm{g} / \mathrm{ml}$ hydrocortisone (Sigma Aldrich), 100ng/ml Cholera toxin (Sigma Aldrich), $10 \mu \mathrm{g} / \mathrm{ml}$ Insulin (Sigma Aldrich), penicillin (100 units $/ \mathrm{ml}$, Sigma Aldrich), and streptomycin $(0.02 \mathrm{mg} / \mathrm{ml}$, Sigma Aldrich) and subcultured according to protocol 1 in reference 17.

\subsection{Sample preparation}

Samples containing fluorescent microspheres were prepared by first making a solution of microspheres with twice the desired concentration. The microsphere solution was then added to a liquid $2 \%$ low-melting point agarose solution (peqGOLD, Peqlab) in a 1:1 ratio and gently mixed. A small bead of agarose/microsphere mixture was then placed in a Petri dish and allowed to set for 10 minutes before being placed on the sample stage and immersed in distilled water. 
MCF-10A cells (see Section 2.3 for culture details) were prepared for suspension firstly by removing cells from the bottom surface of the culture flask by aspirating the growth medium, rinsing the cell monolayer with phosphate buffered saline (PBS, Sigma Aldrich), then adding $2 \mathrm{ml}$ of trypsin solution (0.05\% trypsin, $0.53 \mathrm{mM}$ EDTA, Sigma Aldrich), the excess trypsin was then aspirated before incubating at $37^{\circ} \mathrm{C}$ for 18 minutes. The cells were then suspended in $1 \mathrm{ml}$ PBS and transferred to an Eppendorf tube in which they were gently centrifuged (100x g) for 3 minutes and the supernatant removed. The cells were then fixed with $4 \%(\mathrm{~m} / \mathrm{v})$ paraformaldehyde (PFA, Sigma Aldrich) and incubated at $37^{\circ} \mathrm{C}$ for 15 minutes. The cells were then centrifuged again, the PFA removed and PBS added, centrifuging and aspiration was repeated and finally PBS was added resulting in a fixed cell solution. The cell solution was then added to the $2 \%$ agarose solution as described above.

\section{RESULTS AND DISCUSSION}

\subsection{Calibration}

The theoretical resolution of our light sheet microscope was predicted to be 770nm both laterally and axially with a FoV over which the axial resolution is maintained of approximately $16 \mu \mathrm{m}$. The resolution was tested experimentally by preparing a dilute sample containing sub-diffraction limited polymer microspheres (Duke Scientific R600, 0.6 $\mu \mathrm{m}$ diameter) and imaging one bead at the beam waist. Figure 2 shows $x-y$ and $x-z$ cross sections of a microsphere and their corresponding intensity profiles.

The profiles in Fig. 2(c) and 2(d) were interpolated and the resolution estimated by the full width half maximum (FWHM) values. This gave a lateral resolution (x- and y-axis) of $(780 \pm 30) \mathrm{nm}$ and an axial resolution (z-axis) of (750 \pm 50) $\mathrm{nm}$. This was in agreement with the theoretical values.
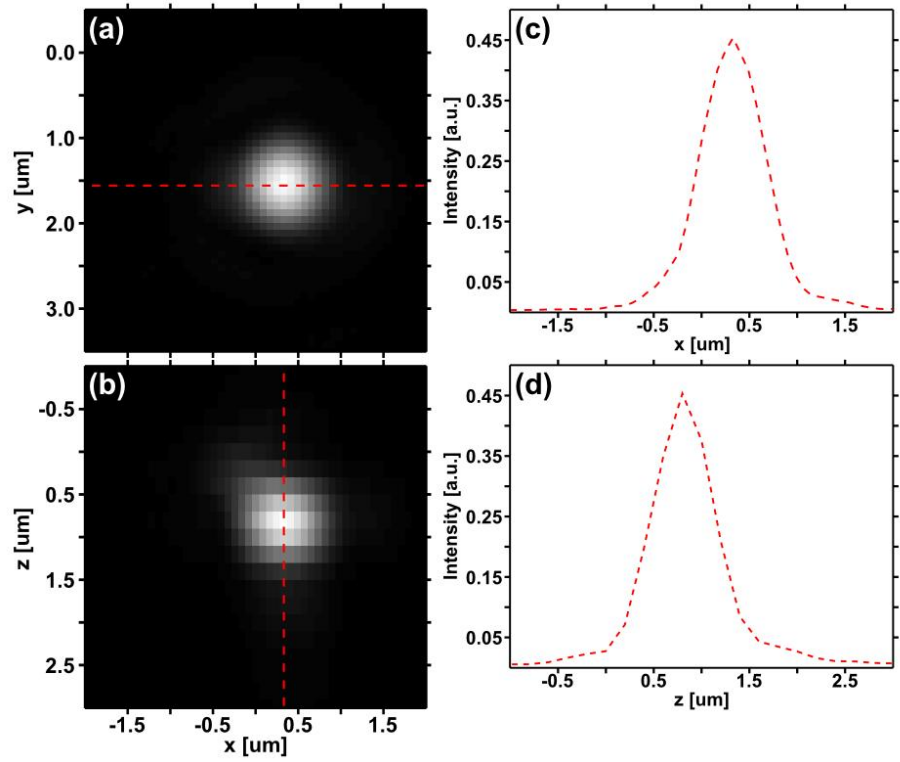

Figure 2. $x-y(a)$ and $x-z$ (b) sections of an LST image of a $600 \mathrm{~nm}$ polymer microsphere located near the beam waist $([x, y, z]=[0,0,0])$ of a Gaussian light sheet. Intensity profiles $(c, d)$ along the dotted lines in (a) and (b) respectively. 


\subsection{Field of view extension with Bessel light sheet illumination}

When a high NA is used for light sheet illumination, the FoV over which the light sheet thickness remains uniform is very small due to rapid divergence of the light sheet and the benefits of optical sectioning are lost. To overcome this in LSFM, propagation invariant Bessel beams have been used for illumination. Bessel beams which remain propagation invariant over some distance can be created by taking the Fourier transform of a thin annular aperture, where we define $R$, the outer radius of the aperture and $R-\Delta R$, the inner radius of the aperture where $\Delta R$ is the width of the annular ring. The propagation invariant region is inversely proportional to the annulus width so we consider two realizations of the Bessel beam, termed Bessel10 and Bessel5, referring to the ratio $\beta=\Delta R / R=0.10$ and 0.05 respectively where Bessel10 has a propagation invariant length of approximately $42 \mu \mathrm{m}$ and Bessel5 remains propagation invariant for twice this length. Both these Bessel beams were holographically generated using the SLM and used to image a sample of $0.6 \mu \mathrm{m}$ diameter polymer microspheres.

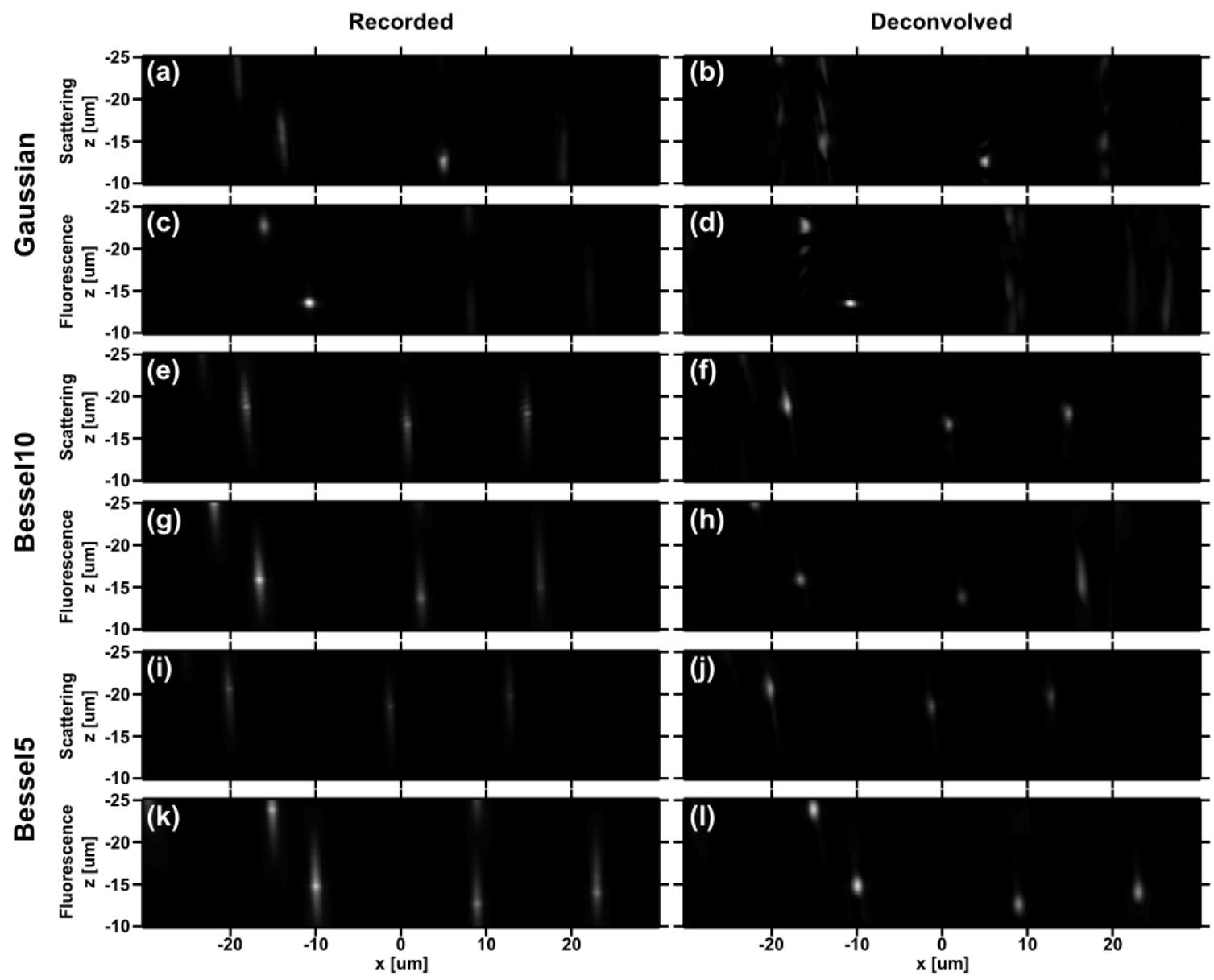

Figure 3. Experimental data showing y-axis maximum intensity projections for both scattering and fluorescence measurements for various light sheet types of the same sample of $600 \mathrm{~nm}$ red fluorescent polymer microspheres. Recorded data is shown in the left hand column, the second column shows the same data after deconvolution. (a,b) Scattering and (c,d) fluorescence measurements acquired using a Gaussian light sheet. (e,f) Scattering and (g,h) fluorescence measurements acquired using a Bessel10 light sheet. (i,j) Scattering and (k,l) fluorescence measurements acquired using a Bessel5 light sheet. Some fluorescence images have been shifted in $\mathrm{x}$ and $\mathrm{z}$ to account for sample drift occurring between scattering and fluorescence measurements. 
Figure 3 shows the results of both scattering and fluorescence measurements for comparison. Fig 3(a) shows the rapid loss of axial resolution with propagation away from focus, the associated loss of contrast in the MTF ${ }^{10}$ is observed as strong image artifacts in the deconvolution (Fig 3(b)) of the image which is also seen in the fluorescence measurements (Fig 3(c,d)). When Bessel beam illumination is used (Fig 3(e-l)) the recorded images are more blurred, but they are blurred uniformly and deconvolution restores axial resolution over a larger FoV. Comparison of LST measurements using Bessel10 and Bessel5 illumination agrees well with what is expected from fluorescence measurements; that increasing the propagation invariance, and therefore the FoV, reduces the contrast in the light sheet MTF and therefore lowers the axial resolution that can be achieved across the FoV. The good agreement between LSFM and LST images after deconvolution suggests that convolution of the light sheet with the sample refractive index distribution for image formation in LST is completely analogous to the convolution of the light sheet with the sample fluorophore distribution in LSFM. Due to the low density of microspheres, only weak artifacts are seen outside of the propagation invariant region of the Bessel beams.

\subsection{Demonstration of Bessel beam LST in biological specimen}

A three-dimensional suspension of MCF-10A cells was prepared (see Section 2.4) to demonstrate the application of extended FoV LST by Bessel beam illumination in a biological specimen. Figure 4 shows LST images acquired using Gaussian and Bessel beam illumination. A fluorescence image (Fig 4(a)) was also taken showing RFP expression throughout the cells for comparison. The Gaussian LSFM (Fig 4(a)) and LST (Fig 4(b)) are closely matched. Due to strong artifacts over most of the image, no deconvolved data is shows for the Guassian light sheet. Again, comparison between the data acquired with Bessel10 and Bessel5 light sheets shows a reduction in axial resolution when increasing the FoV. However, comparison between recorded Gaussian (Fig 4(b)) and deconvolved Bessel5 (Fig 4(f)) images shows that the reduced axial resolution over the entire image is less detrimental to overall image quality than an axial resolution that degrades with propagation from focus. LST images in Figure 4 show evidence of sub-cellular details, however, due to lack of specificity, the cellular components observed cannot be identified.

\section{CONCLUSION}

Light sheet fluorescence microscopy has enabled a step-change in imaging for developmental biology as well as other disciplines due to its well suited imaging properties and light sheet tomography is emerging as another potentially disruptive implementation of the light sheet principle that can be readily implemented on any existing light sheet fluorescence microscope. Wavefront shaping techniques have been successfully applied to LSFM to make the technology even more powerful.

We demonstrate the use of propagation invariant Bessel beam light modes for illumination in LST to extend the high resolution field of view of the microscope by a factor 4 with only a small reduction in axial resolution. More advanced Bessel beam light sheet techniques such as those incorporating structured illumination algorithms ${ }^{13,14}$ or confocal line scanning ${ }^{[11]}$ could be used to enhance LST further. Additionally, in situ aberration correction ${ }^{15}$, which was applied here to counteract aberrations present in the microscope optics, may be used at depth or within turbid specimens to counteract sample-induced aberrations as was demonstrated for LSFM ${ }^{18}$.

Through comparison of LST and LSFM images we elucidate the role of light sheet convolution in image formation in LST, which is found to be analogous to its role in LSFM. This important result confirms, not only that Bessel beams may be applied to LST for extending the FoV, but that it should be possible for any wavefront shaping technique successfully applied to LSFM to be translated to LST.

Finally, we demonstrate the applicability of the Bessel beam LST method in a biological specimen, a suspension of fixed cells. Cells are resolved well with the technique and many sub-cellular details appear like speckle within all cells. These sub-cellular details highlight the lack of specificity in LST, however, for sufficiently sparse samples, the lack of specificity is advantageous for rapid, volumetric imaging requiring no special sample preparation. 

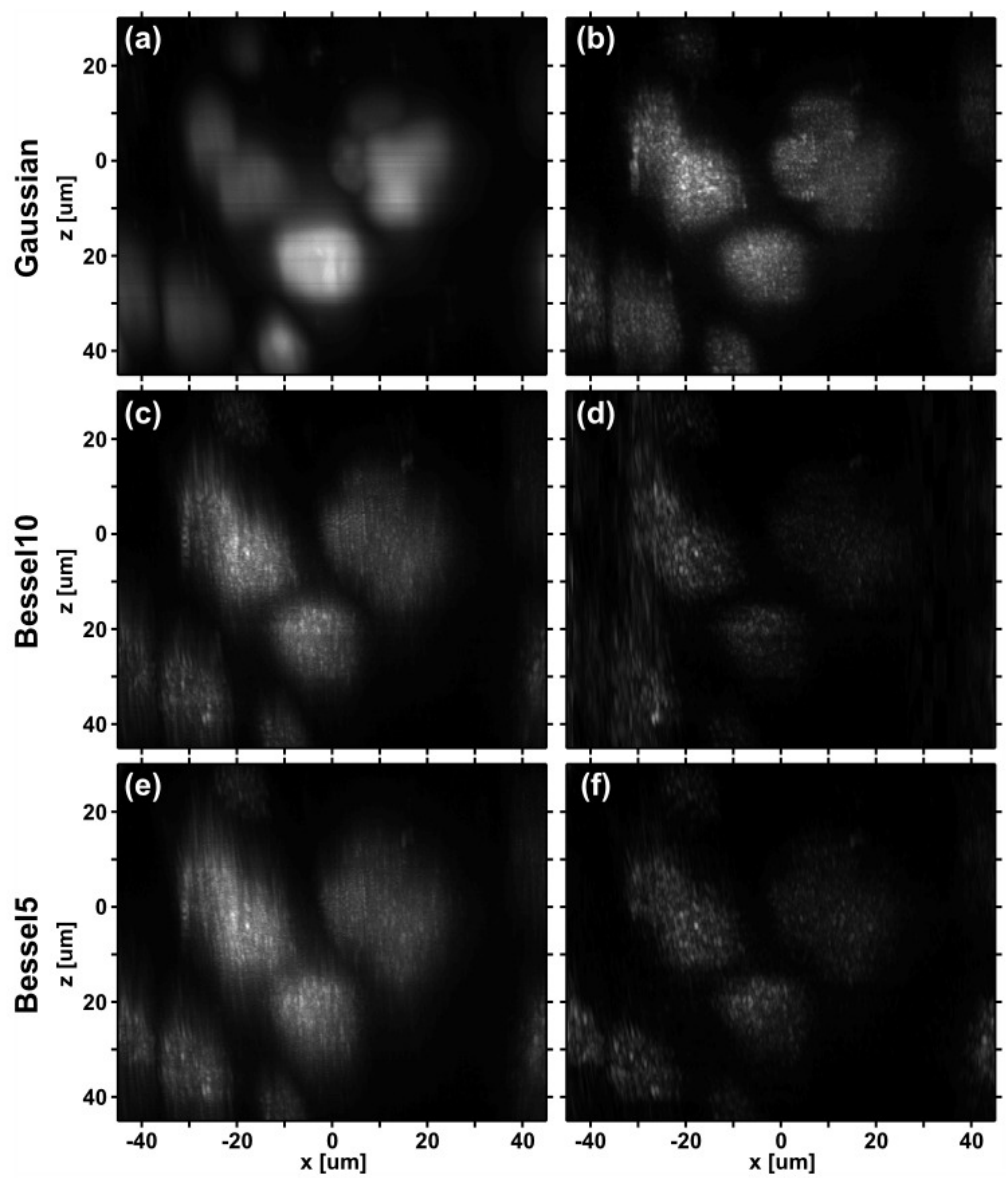

Figure 4. Experimental data showing y-axis maximum intensity projections for scattering measurements for various light sheet types of the same sample of MCF-10A cell suspension. (a) Gaussian light sheet fluorescence measurement for comparison. (b) Recorded data acquired using a Gaussian light sheet. (c,d) Recorded and deconvolved images respectively acquired using a Bessel10 light sheet. (e, f) Recorded and deconvolved images respectively acquired using a Bessel5 light sheet. The horizontal banding in (a) is attributed to laser power fluctuations.

\section{ACKNOWLEDGMENTS}

The project was supported by the UK Engineering and Physical Sciences Research Council, RS MacDonald Charitable Trust, SULSA, and the St. Andrews 600th anniversary BRAINS appeal. K. D. is a Royal Society Wolfson Merit Award holder.

\section{REFERENCES}

[1] Voie, A.H., Burns, D.H., and Spelman, F.A., "Orthogonal-plane fluorescence optical sectioning: threedimensional imaging of macroscopic biological specimens", J. Microsc. 170, 229-236 (1993)

[2] Keller, P.J. et al, "Reconstruction of Zebrafish Early Embryonic Development by Scanned Light Sheet Microscopy", Science 322(5904), 1065-1069 (2008) 
[3] Huisken, J. \& Stainier, D.Y.R., "Selective plane illumination microscopy techniques in developmental biology", Development 136, 1963-1975 (2009)

[4] Zanacchi, F.C. et al, "Live-cell 3D super-resolution imaging in thick biological samples", Nature Methods 8, 1047-1049 (2011)

[5] Swoger, J. et al, "4D retrospective lineage tracing using SPIM for zebrafish organogenesis studies", J. Biophotonics 4(1-2), 122-134 (2011)

[6] Tomer, R. et al, "Quantitative high-speed imaging of entire developing embryos with simultaneous multiview light-sheet microscopy", Nature Methods 9, $755-763$ (2012)

[7] Maizel, A. et al, "High-resolution live imaging of plant growth in near physiological bright conditions using light sheet fluorescence microscopy", Plant J. 68(2), 377-385 (2011)

[8] Yang, Z.Y. et al, "Light Sheet Tomography (LST) for in situ imaging of plant roots", Optics Express 21(14), 16239-16247 (2013)

[9] Jemielita, M. et al, "Comparing phototoxicity during the development of a zebrafish craniofacial bone using confocal and light sheet fluorescence microscopy techniques", J. Biophotonics 6(11-12), 920-928 (2013)

[10] Vettenburg, T., Dalgarno, H.I.C., Čižmár, T., Dholakia, K., "The role of propagation invariant light modes in single and multi-photon imaging", Proc. SPIE 8637 (2013)

[11] Fahrbach, F.O. \& Rohrbach, A., "Propagation stability of self-reconstructing Bessel beams enables contrastenhanced imaging in thick media", Nature Communications 3, 632 (2012)

[12]Fahrbach, F.O. \& Rohrbach, A., "A line scanned light-sheet microscope with phase shaped self-reconstructing beams", Optics Express 18(23), 24229-24244 (2010)

[13] Planchon, T.A. et al, "Rapid three-dimensional isotropic imaging of living cells using Bessel beam plane illumination", Nature Methods 8, 417-423 (2011)

[14] Gao, L. et al, "Noninvasive Imaging beyond the Diffraction Limit of 3D Dynamics in Thickly Fluorescent Specimens", Cell 151(6), 1370-1385 (2012)

[15] Čižmár, T., Mazilu, M., Dholakia, K., "In situ wavefront correction and its application to micromanipulation", Nature Photonics 4, 388-394 (2010)

[16] Gonzalez, R.C. \& Woods, R.E., [Digital Image Processing, 3rd ed.], Prentice Hall (2007)

[17] Debnath, J., Muthuswamy, S.K., \& Brugge, J.S., "Morphogenesis and oncogenesis of MCF-10A mammary epithelial acini grown in three-dimensional basement membrane cultures", Methods 30(3), 256-268 (2003)

[18] Dalgarno, H.I.C. et al, "Wavefront corrected light sheet microscopy in turbid media", Appl. Phys. Lett. 100 (2012) 